\title{
The Gaussian Radial Basis Function Method for Plasma Kinetic Theory
}

\author{
E. Hirvijoki ${ }^{\mathrm{a}}$, J. Candy ${ }^{\mathrm{b}}$, E. Belli ${ }^{\mathrm{b}}$, O. Embréus ${ }^{\mathrm{a}}$
}

${ }^{a}$ Department of Applied Physics, Chalmers University of Technology, SE-41296 Gothenburg, SWEDEN
${ }^{b}$ General Atomics, PO Box 85608, San Diego, CA 92186-5608, USA

\begin{abstract}
Description of a magnetized plasma involves the Vlasov equation supplemented with the non-linear Fokker-Planck collision operator. For non-Maxwellian distributions, the collision operator, however, is difficult to compute. In this letter, we introduce Gaussian Radial Basis Functions (RBFs) to discretize the velocity space of the entire kinetic system, and give the corresponding analytical expressions for the Vlasov and collision operator. Outlining the general theory, we also highlight the connection to plasma fluid theories, and give 2D and 3D numerical solutions of the non-linear Fokker-Planck equation. Applications are anticipated in both astrophysical and laboratory plasmas.
\end{abstract}

Keywords: Coulomb collisions, non-linear, kinetic theory, Fokker-Planck

PACS: 52.25.Dg, 02.60.Nm, 02.70.Jn, 52.65.Ff

\section{Motivation}

A fundamental macroscopic description of a magnetized plasma is the Vlasov equation supplemented by the non-linear inverse-square force Fokker-planck collision operator [1]

$$
\frac{\partial f_{a}}{\partial t}+\mathbf{v} \cdot \nabla f_{a}+\frac{e_{a}}{m_{a}}(\mathbf{E}+\mathbf{v} \times \mathbf{B}) \frac{\partial f_{a}}{\partial \mathbf{v}}=\sum_{b} C_{a b}\left(f_{a}, f_{b}\right),
$$

where $f_{a}$ is the distribution of species $a$ with charge $e_{a}$ and mass $m_{a}$. The electric and magnetic fields depend on the distribution $f_{a}$ through Maxwell's equations. This model assumes a statistical description of Coulomb interaction in the limit of smallangle scattering, with the changes in $f_{a}$ due to collisions with species $b$ described by

$$
C_{a b}=\frac{\partial}{\partial \mathbf{v}} \cdot\left[\mathbf{A}_{a b} f_{a}+\frac{\partial}{\partial \mathbf{v}} \cdot\left(\mathbb{D}_{a b} f_{a}\right)\right] .
$$

The friction and diffusion coefficients are given by the expressions

$$
\mathbf{A}_{a b}=L_{a b}\left(1+\frac{m_{a}}{m_{b}}\right) \frac{\partial \varphi_{b}}{\partial \mathbf{v}}, \quad \mathbb{D}_{a b}=-L^{a b} \frac{\partial^{2} \psi_{b}}{\partial \mathbf{v} \partial \mathbf{v}},
$$

where $L_{a b}=\left(e_{a} e_{b} / m_{a} \varepsilon_{0}\right)^{2} \ln \Lambda_{a b}$ and $\ln \Lambda_{a b}$ is the Coulomb logarithm which respresents a physical cut-off for small-angle collisions. The Rosenbluth potentials appearing in the friction and diffusion coefficients are weighted integrals of the distribution function

$$
\begin{aligned}
& \varphi_{b}(\mathbf{x}, \mathbf{v}, t)=-\frac{1}{4 \pi} \int d \mathbf{v}^{\prime} f_{b}\left(\mathbf{x}, \mathbf{v}^{\prime}, t\right) \frac{1}{\left|\mathbf{v}-\mathbf{v}^{\prime}\right|} \\
& \psi_{b}(\mathbf{x}, \mathbf{v}, t)=-\frac{1}{8 \pi} \int d \mathbf{v}^{\prime} f_{b}\left(\mathbf{x}, \mathbf{v}^{\prime}, t\right)\left|\mathbf{v}-\mathbf{v}^{\prime}\right|
\end{aligned}
$$

Email address: eero.hirvijoki@chalmers.se (E. Hirvijoki) and they satisfy the velocity-space Poisson equations $\nabla_{\mathbf{v}}^{2} \psi_{b}=$ $\varphi_{b}$ and $\nabla_{\mathbf{v}}^{2} \varphi_{b}=f_{b}$. Expressed in terms of the potential functions, the Fokker-Planck collision operator is

$$
C_{a b}=L_{a b}\left[\frac{m_{a}}{m_{b}} f_{a} f_{b}+\mu_{a b} \frac{\partial \varphi_{b}}{\partial \mathbf{v}} \cdot \frac{\partial f_{a}}{\partial \mathbf{v}}-\frac{\partial^{2} \psi_{b}}{\partial \mathbf{v} \partial \mathbf{v}}: \frac{\partial^{2} f_{a}}{\partial \mathbf{v} \partial \mathbf{v}}\right],
$$

where $\mu_{a b}=m_{a} / m_{b}-1$. A common approach for numerical evaluation of $C_{a b}$ follows a two-phase method where one first inverts the velocity-space Laplacian operators and then directly evaluates the collision operator. Boundary conditions for the Poisson equations can be obtained by limiting the solution to a sub-space and evaluating the expressions at the boundary using a multipole expansion of the potentials [2], or by imposing the free-space solution outside the sub-space [3]. Another sophisticated approach is based on fast spectral decomposition as described in Ref. [4].

In this letter, we propose a new approach using a mesh-free shifted-Maxwellian representation which is intuitively appealing and straightforward to implement. The solution thus obtained is $C^{\infty}$ smooth, extends to $v \rightarrow \infty$, and allows compact representation of any interesting macroscopic quantity (number, momentum, energy density, and so on). The Letter is organized as follows: In Sec. 2 we present the idea for using Gaussian radial basis functions to solve the kinetic equation. The numerical implementation is described in Sec. 3 and simulations in 2D and 3D velocity space are presented in Sec. 4. Finally, we discuss and summarize our results in Sections 5 and 6.

\section{The Gaussian RBF Method}

To solve the kinetic equation, Eq. (1), we write the total distribution as a finite sum of shifted Maxwellians

$$
f_{a}(\mathbf{x}, \mathbf{v}, t)=\sum_{i} w_{a}^{i}(\mathbf{x}, t) F_{a}^{i}(\mathbf{x}, \mathbf{v})
$$

July 15, 2015 
where each Maxwellian, $F_{a}^{i}=\left(\gamma_{a}^{i} / \pi\right)^{3 / 2} \exp \left[-\gamma_{a}^{i}\left(\mathbf{v}-\mathbf{v}_{a}^{i}\right)^{2}\right]$, is normalized to unity and the weights $w_{a}^{i}$ allowed to evolve in time. The width parameters $\gamma_{a}^{i}$ and mean velocities $\mathbf{v}_{a}^{i}$ can be arbitrary functions of position. The shifted Maxwellians are nothing other than Gaussian Radial Basis functions (RBFs) which have found numerous applications in applied mathematics - in particular for the construction of neural networks [5]. For compactness, in what follows we will retain the spatial dependence of all quantities but will not write the dependence explicitly.

The potential functions then take the form

$$
\varphi_{a}(\mathbf{v}, t)=\sum_{i} w_{a}^{i}(t) \varphi_{a}^{i}(\mathbf{v}), \quad \psi_{a}(\mathbf{v}, t)=\sum_{i} w_{a}^{i}(t) \psi_{a}^{i}(\mathbf{v})
$$

where the Gaussian RBF potentials $\varphi_{a}^{i}=-\sqrt{\gamma_{a}^{i}} \Phi\left(\sqrt{\gamma_{a}^{i}} \mid \mathbf{v}-\right.$ $\left.\mathbf{v}_{a}^{i} \mid\right) /(4 \pi)$ and $\psi_{a}^{i}=-\left(1 / \sqrt{\gamma_{a}^{i}}\right) \Psi\left(\sqrt{\gamma_{a}^{i}}\left|\mathbf{v}-\mathbf{v}_{a}^{i}\right|\right) /(8 \pi)$ are defined in terms of the functions $\Phi(s)=\operatorname{erf}(s) / s$ and $\Psi(s)=$ $[s+1 /(2 s)] \operatorname{erf}(s)+\exp \left(-s^{2}\right) / \sqrt{\pi}$, where $\operatorname{erf}(s)$ is the error function. We thus find a simple bilinear expression for the complete non-linear operator

$$
C_{a b}=\sum_{k, \ell} w_{a}^{k}(t) w_{b}^{\ell}(t) C_{a b}^{k \ell}(\mathbf{v}),
$$

where the Gaussian RBF collision tensor is

$$
C_{a b}^{k \ell}=L_{a b}\left[\frac{m_{a}}{m_{b}} F_{a}^{k} F_{b}^{\ell}+\mu_{a b} \frac{\partial \varphi_{a}^{k}}{\partial \mathbf{v}} \cdot \frac{\partial F_{b}^{\ell}}{\partial \mathbf{v}}-\frac{\partial^{2} \psi_{a}^{k}}{\partial \mathbf{v} \partial \mathbf{v}}: \frac{\partial^{2} F_{b}^{\ell}}{\partial \mathbf{v} \partial \mathbf{v}}\right],
$$

such that $C_{a a}^{k k}(\mathbf{v})=0$. As we have analytical expressions for $F_{a}^{i}(\mathbf{v}), \varphi_{a}^{i}(\mathbf{v})$, and $\psi_{a}^{i}(\mathbf{v})$, the tensor $C_{a b}^{k \ell}(\mathbf{v})$ is easy to implement and fast to evaluate at any point in velocity space.

In problems with azimuthal symmetry, a 2D RBF scheme can be developed with axisymmetric ring-like RBF-basis:

$$
F_{a}^{i}=\left(\gamma_{a}^{i} / \pi\right)^{3 / 2} I_{0}\left(2 \gamma_{a}^{i} v_{a, \perp}^{i} v_{\perp}\right) e^{-\gamma_{a}^{i}\left(v_{\|}-v_{a, \|}^{i}\right)^{2}-\gamma_{a}^{i}\left(v_{\perp}^{2}+\left(v_{a, \perp}^{i}\right)^{2}\right)},
$$

where $I_{0}$ is the order-zero modified Bessel function of the first kind and $\left(v_{\perp}, v_{\|}\right)$are the cylindrical velocity-space coordinates. Although explicit expressions for axisymmetric RBF potentials $\varphi_{i}$ and $\psi_{i}$ are not available in a closed form, they can be evaluated numerically to machine precision at any requested point.

\section{Collocation Options}

We describe two different methods for obtaining an ordinary differential equation for the time evolution of weights: the Galerkin and the center-collocation projections. In the Galerkin method, the kinetic equation - already expanded in the RBF basis - is multiplied by each individual basis function and then integrated over the entire domain. Conversely, in the center-collocation method, the kinetic equation is evaluated at the center of each RBF. Both methods yield $N$ equations for the $N$ RBF weights, $w_{a}^{i}$, and result in a differential equation for the weights that can be written in a matrix form.

For the moment, let us illustrate the method by considering the spatially homogeneous case with no electromagnetic fields. Then, the matrix equation is

$$
\sum_{j} M_{a}^{i j} \frac{\partial w_{a}^{j}}{\partial t}=\sum_{b, k, \ell} w_{a}^{k}(t) w_{b}^{\ell}(t) C_{a b}^{i k \ell} \quad \forall \quad i \in 1,2,3, \ldots,
$$

In the Galerkin projection (GP), the matrix $M_{i j}$ is symmetric, typically diagonally dominant, and given by the expression

$$
\left(M_{a}^{i j}\right)_{\mathrm{GP}}=\left(\frac{\gamma_{a}^{i} \gamma_{a}^{j}}{\pi\left(\gamma_{a}^{i}+\gamma_{a}^{j}\right)}\right)^{3 / 2} \exp \left[-\frac{\gamma_{a}^{i} \gamma_{a}^{j}}{\gamma_{a}^{i}+\gamma_{a}^{j}}\left(\mathbf{v}_{a}^{i}-\mathbf{v}_{a}^{j}\right)^{2}\right],
$$

whereas in the center-collocation (CC) method, the matrix $M_{a}^{i j}$ is no longer necessarily symmetric, but can still be dominated by the diagonal components:

$$
\left(M_{a}^{i j}\right)_{\mathrm{CC}} \equiv F_{a}^{j}\left(\mathbf{v}_{a}^{i}\right)=\left(\gamma_{a}^{j} / \pi\right)^{3 / 2} \exp \left[-\gamma_{a}^{j}\left(\mathbf{v}_{a}^{i}-\mathbf{v}_{a}^{j}\right)^{2}\right]
$$

On the right-hand-side of Eq. (2), the tensor $C_{a b}^{i k \ell}$ becomes

$$
\left(C_{a b}^{i k \ell}\right)_{\mathrm{GP}}=\int d \mathbf{v} F_{a}^{i}(\mathbf{v}) C_{a b}^{k \ell}(\mathbf{v}), \quad\left(C_{a b}^{i k \ell}\right)_{\mathrm{CC}}=C_{a b}^{k \ell}\left(\mathbf{v}_{i}\right),
$$

for the Galerkin and center-collocation, respectively. Obtaining the center-collocation tensor $\left(C_{a b}^{i k \ell}\right)_{\mathrm{CC}}$ is merely a matter of evaluating the RBF tensor $C_{a b}^{k \ell}(\mathbf{v})$ at the collocation points. Evaluation of the tensor $\left(C_{a b}^{i k \ell}\right)_{\text {GP }}$ for the Galerkin projection is somewhat more complicated, although the result may be potentially be more accurate or robust.

Nevertheless, to maintain simplicity, we focus hereafter on the center collocation-method and omit the CC subscript for brevity. In this case the RBF equations for the full non-linear system become

$$
\sum_{j} M_{a}^{i j} \mathcal{L}_{a}^{i j} w_{a}^{j}=\sum_{b, k, \ell} w_{a}^{k} w_{b}^{\ell} C_{a b}^{i k \ell}, \quad \forall \quad i \in 1,2,3, \ldots,
$$

where the operator $\mathcal{L}$

$$
\mathcal{L}_{a}^{i j} \doteq \frac{\partial}{\partial t}+\mathbf{v}_{a}^{i} \cdot \nabla+\frac{e_{a}}{\sigma_{a}^{j}}\left[\left(\mathbf{v}_{a}^{j}-\mathbf{v}_{a}^{i}\right) \cdot \mathbf{E}+\left(\mathbf{v}_{a}^{j} \times \mathbf{v}_{a}^{i}\right) \cdot \mathbf{B}\right]
$$

retains the familiar appearance of the Vlasov operator even though the velocity-space has been completely removed from the problem. Note that $\mathcal{L}$ depends explicitly on species $a$ and implicitly on $b$ via the Maxwell equations. In $\mathcal{L}$ we have defined the temperature-like parameter $\sigma_{a}^{i}=m_{a} /\left(2 \gamma_{a}^{i}\right)$ and also dropped some additional terms that arise if the parameters $\gamma_{a}^{i}$ and $\mathbf{v}_{a}^{i}$ depend on position. The RBF-kinetic equation (3) describes collisional fluid-like evolution of the weights in time and space. One physically appealing feature of the RBF expansion is that familiar expressions for macroscopic fluid moments retain their intuitive form:

$$
\begin{aligned}
\text { Density } n_{a} & =\sum_{i} w_{a}^{i} \\
\text { Velocity } n_{a} \mathbf{V}_{a} & =\sum_{i} w_{a}^{i} \mathbf{v}_{a}^{i} \\
\text { Temperature } \frac{3}{2} n_{a} T_{a} & =\sum_{i} w_{a}^{i}\left[\frac{3}{2} \sigma_{a}^{i}+\frac{1}{2} m_{a}\left(\mathbf{v}_{a}^{i}-\mathbf{V}_{a}\right)^{2}\right] \\
\text { Momentum flux tensor } \quad \mathbf{\Pi}_{a} & =\sum_{i} w_{a}^{i} m_{a}\left[\sigma_{a}^{i} \mathbb{I}+\mathbf{v}_{a}^{i} \mathbf{v}_{a}^{i}\right] \\
\text { Energy flux } \quad \mathbf{Q}_{a} & =\sum_{i} w_{a}^{i} \mathbf{v}_{a}^{i}\left[\frac{5}{2} \sigma_{a}^{i}+\frac{1}{2} m_{a}\left(v_{a}^{i}\right)^{2}\right],
\end{aligned}
$$


Another is that also the Maxwell's equations can be written compactly:

$$
\begin{aligned}
\nabla \cdot \mathbf{E} & =\left(1 / \varepsilon_{0}\right) \sum_{a} e_{a} n_{a}=\left(1 / \varepsilon_{0}\right) \sum_{a, i} e_{a} w_{a}^{i} \\
\nabla \times \mathbf{B} & =\mu_{0} \sum_{a} e_{a} n_{a} \mathbf{V}_{a}=\mu_{0} \sum_{a, i} e_{a} w_{a}^{i} \mathbf{v}_{a}^{i}
\end{aligned}
$$

where for simplicity we have neglected the displacement current. In contrast to the standard fluid approach, where calculation of the higher-order velocity-space moments becomes increasingly cumbersome, the RBF-kinetic approach offers a straightforward and intuitive alternative by describing the velocity space with Gaussian functions that naturally appear in the kinetic theory as thermodynamic equilibrium solutions. Moreover, so long as the RBF spacing is equal to about one e-folding length, the problem remains well-conditioned even for hundreds or thousands of basis functions.

It should also be evident that the Gaussian RBF method is well-suited to linearized models. The linearized collision operator in this case is just the sum of $C^{0 i}$ and $C^{i 0}$; namely, the first row and column of the non-linear collision tensor. Because the method is mesh-free, it is also natually suited to multi-species plasmas, unlike contemporary algorithms that are based on a velocity mesh [6].

\section{Non-Linear Simulations in $2 D$ and 3D}

To assess the viability of the Gaussian RBF approach, we study a single species plasma using a uniform grid for the collocation points $\mathbf{v}_{a}^{i}$ and a fixed value $\gamma_{a}^{i}=\gamma$ for all RBFs. We also normalize time with the collision time, i.e., $\tau=L_{a a} t$. Our problem is thus to solve the non-linear single species FokkerPlanck equation

$$
\frac{\partial f}{\partial \tau}=f^{2}-\frac{\partial^{2} \psi}{\partial \mathbf{v} \partial \mathbf{v}}: \frac{\partial^{2} f}{\partial \mathbf{v} \partial \mathbf{v}} \equiv C[f]
$$

We emphasize that this is a highly nontrivial numerical problem. First, we test how well the discretization preserves a (stationary) Maxwellian equilibrium state, where the temperature of the equilibrium is fixed and much warmer than the individual RBFs. For the analytic equilibrium we choose $f_{M}=$ $(\beta / \pi)^{3 / 2} \exp \left[-\beta \mathbf{v}^{2}\right]$, with $\beta=1 / 4$ and arrange the collocation points into uniform rectilinear grids in spaces $\left[v_{x}, v_{y}, v_{z}\right] \in$ $[-6.5,6.5] \times[-6.5,6.5] \times[-6.5,6.5]$ and $\left[v_{\|}, v_{\perp}\right] \in[-6.5,6.5] \times$ $[0,6.5]$ for the full $3 \mathrm{D}$ and axisymmetric $2 \mathrm{D}$ methods respectively. The equilibrium is projected onto the RBF basis to obtain the numerical equivalent of the steady state distribution function. Then the collision operator in Eq. (4) is evaluated for an increasing number of collocation points and different values of $\gamma$. As an error metric, we choose $\max \{C[f] / f\}$, presented in Fig. 1. This test is sensitive to the error both in the region interior to the RBF collocation centers, as well as to the region beyond them. As is evident from Fig. 1, the maximum of the global value decreases when the number of collocation points is increased indicating that a numerical equivalent to the analytical steady-state can be reached.

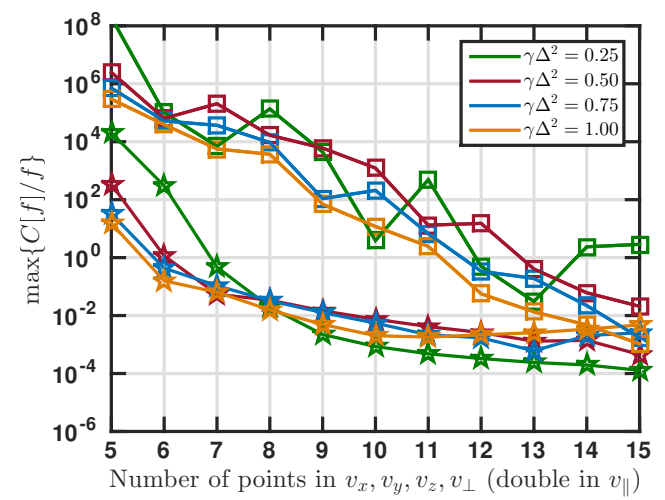

Figure 1: Convergence of a steady-state collision operator. The lines with square (star) markers represent the $3 \mathrm{D}$ (axisymmetric 2D) solutions and $\Delta$ is the distance between adjacent collocation points.

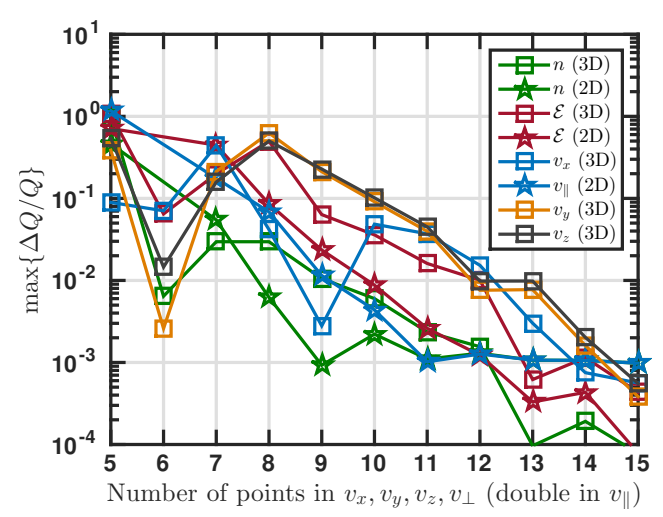

Figure 2: Convergence of the velocity space moments in a relaxation study. Here $\gamma \Delta^{2}=0.5$ was used and $\Delta$ is the distance between adjacent collocation points.

Next, we follow the relaxation of a non-trivial distribution function to the equilibrium state and track the conservation of number, momentum, and energy densities. The initial state of the distribution function is set to $f(\mathbf{v}, \tau=0)=$ $\sum_{i=1}^{2} e^{-\beta_{i}\left(\mathbf{v}-\mathbf{v}_{i}\right)^{2}}$, where $\beta_{i}=1 / 5, \mathbf{v}_{1}=(3,0,0), \mathbf{v}_{2}=(-3,0,0)$ for the $\left(v_{x}, v_{y}, v_{z}\right)$ components respectively. The initial state is thus axisymmetric with respect to the axis $v_{x}$ and the the axisymmetric setting is thus chosen to be $\left(v_{\|}=v_{x}, v_{\perp}^{2}=\right.$ $\left.v_{z}^{2}+v_{y}^{2}\right)$. The collocation points are chosen uniformly in the regions $\left[v_{x}, v_{y}, v_{z}\right] \in[-8,8] \times[-8,8] \times[-8,8]$ and $\left[v_{\|}, v_{\perp}\right] \in$ $[-12,12] \times[0,12]$, the initial state projected to the Gaussian RBF basis to get initial values for the weights, and the weights then evolved in time according to the Eq. (2) using a standard fourth-order Runge-Kutta method. From the time-evolution of the weights, we have calculated the evolution of the velocityspace moments, and recorded their maximal deviations from the initial values during the simulation. The results are illustrated in Fig. 2, where one observes good conservation of number $(n)$, momentum (velocity components $v_{x}, v_{y}$, and $v_{z}$ ), and energy $(\mathcal{E})$ densities for both full $3 \mathrm{D}$, and axisymmetric $2 \mathrm{D}$ solvers.

Even better conservation properties can be reached by embedding the conservation laws into the time-evolution of the 


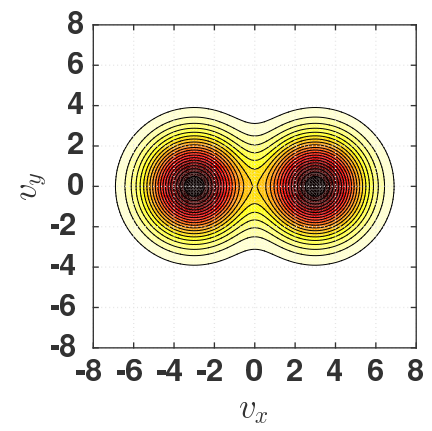

(a) $\quad \tau=0$

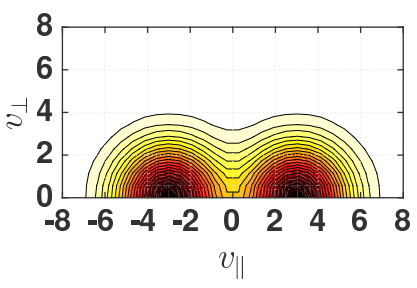

(e) $\quad \tau=0$

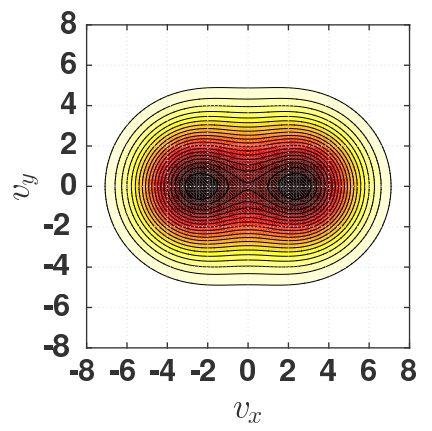

(b) $\quad \tau=3$

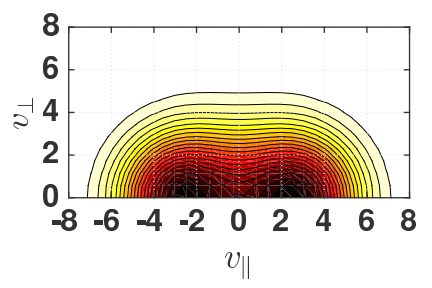

(f) $\quad \tau=3$

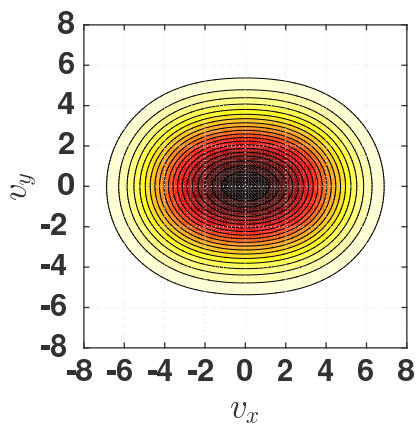

(c) $\quad \tau=6$

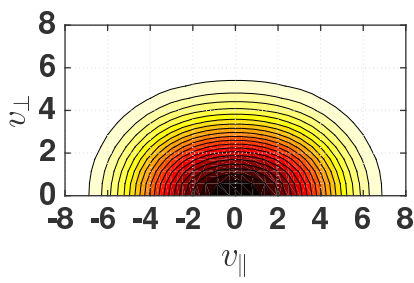

(g) $\quad \tau=6$

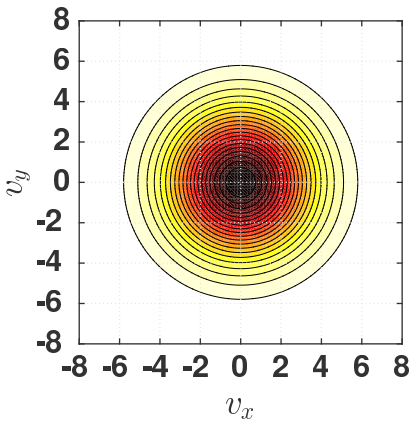

(d) $\quad \tau=40$

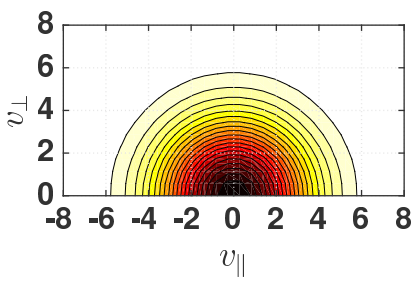

(h) $\quad \tau=40$

Figure 3: Relaxation of an axisymmetric initial state towards spherically symmetric equilibrium state from the $3 \mathrm{D}(\mathrm{a}, \mathrm{b}, \mathrm{c}, \mathrm{d})$ and $2 \mathrm{D}(\mathrm{e}, \mathrm{f}, \mathrm{g}, \mathrm{h})$ solvers using $15 \times 15 \times 15$ $3 \mathrm{D}$ RBFs and $30 \times 15$ axisymmetric RBFs.

weights. For example, replacing one row in the matrix $\left(M^{i j}\right)_{\mathrm{CC}}$ with ones (unity moments of the basis functions) and setting the corresponding element in $\left(C^{i k l}\right)_{C C}$ to zero neglects information from one of the basis nodes but improves density conservation. With this approach we observed, e.g., density conservation up to 12 digits without relevant increase in the computation time.

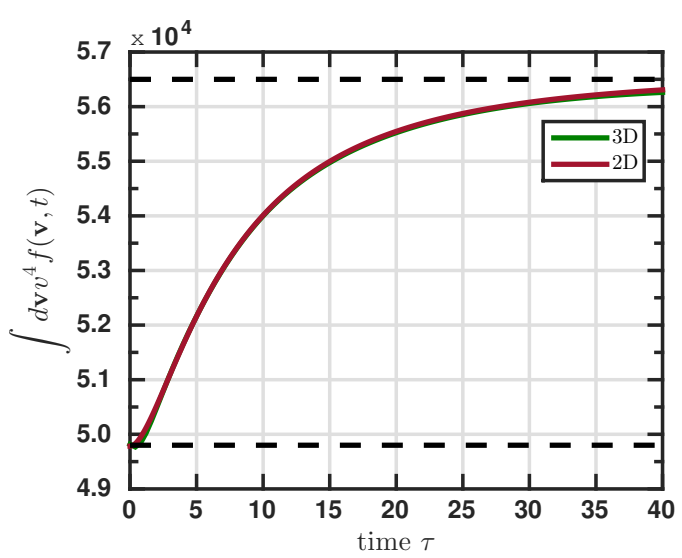

Figure 4: Time evolution of the $v^{4}$-moment during the relaxation study (the almost overlapping red and green curves). The lower (upper) dashed line represents the analytical initial (equilibrium) values.

The time evolution of the distribution in the conservation study using $15^{3} 3 \mathrm{D}$ RBFs and $30 \times 15$ axisymmetric RBFs is illustrated in Fig. 3 with slices in $\left(v_{x}, v_{y}\right)$-plane for the full 3D solver and in $\left(v_{\|}, v_{\perp}\right)$-plane for the 2D axisymmetric solver. The time slices are chosen for the initial state $(\tau=0)$ in Figs. 3(a) and 3(e), for the beginning of the relaxation process
( $\tau=3$ ) in Figs. 3(b) and 3(f), for the merging phase towards the equilibrium $(\tau=6)$ in Figs. 3(c) and 3(g), and for the final state $(\tau=40)$ where the distribution function is close to spherical symmetry and equilibrium in Figs. 3(d) and 3(h). Both the 2D and $3 \mathrm{D}$ solvers qualitatively describe the same solution to the initially axisymmetric problem and preserve the initial symmetry. As a more quantitative measure, we also follow the time evolution of the $v^{4}$-moment of the distribution function which is not a conserved quantity. From the analytical initial state one can calculate the value to be $4.98 \times 10^{4}$ and for the corresponding analytical equilibrium state the value is $5.65 \times 10^{4}$. The time evolution of $v^{4}$-moment from the numerical calculation is shown in Fig. 4. As is clear, the 2D and 3D solutions agree with each other and also confirm our claim that the solution in Figs. 3(d) and 3(h) is close to the analytical equilibrium state.

\section{Discussion}

The tests described above show that the RBF approach is able to accurately treat non-linear collisional relaxation in both 2D and 3D velocity space. Although a thorough comparison and benchmark against other solvers is outside the scope of the present study, we note that the conservation properties of the new method are at least comparable to the approach described in Ref. [2]. In particular, for non-linear axisymmetric relaxation in $2 \mathrm{D}$, the new scheme gives a relative error in the energy moment of roughly $10^{-4}$ for $30 \times 15$ velocity space RBFs. In Fig. 4 of Ref. [2], where a similar relaxation study starting with a slightly less non-linear initial state is shown, a relative error of $10^{-3}$ is found for $128 \times 128$ grid points.

We have also observed that conservation error is sensitive to both the size of the domain covered by the RBF collocation 
points and the choice of $\gamma \Delta^{2}$ (inverse flatness of the RBFs). First, reducing the domain the collocation points cover introduces a residual error because a larger portion of the velocity space is neglected. This affects conservation properties as illustrated in Fig. 5 for density. Second, the choice of $\gamma \Delta^{2}$ affects the condition number $\kappa\left(M^{i j}\right)$ of the RBF collocation matrix, as illustrated in Fig. 6. The condition number then affects the accuracy of the initial projection to the RBF basis and the time evolution since Eq. (3) is multiplied by the inverse of the RBF matrix during every explicit Runge-Kutta step.

The condition number issue is well-known in the context of RBF interpolation (see Ref. [7] and references therein). Although increasing flatness leads to interpolants which are more accurate, it also leads to large condition number of the RBF matrix and corresponding loss of accuracy. In our tests, we observed the condition number and the residual error to have a combined effect. Expanding the collocation domain while keeping the absolute adjacent distance of the RBF collocation points equal improves the conservation properties: In Fig. 5 the yellow curve at $N=15$ and the green curve at $N=11$ both correspond to $\Delta=1$ but the density conservation is better by two orders of magnitude with the larger domain. Carrying out similar sensitivity test for the collocation domain as in Fig. 5 but for different $\gamma \Delta^{2}$ showed overall degradation of the conservation properties for smaller $\gamma \Delta^{2}$, as one would expect due to increase in the condition number.

It is not surprising that the residual error is important. It is a common source of error in methods that have to deal with infinite domains: spectral methods may suffer from aliasing effects due to periodic basis functions (see Ref. [4] and References therein) and finite difference or element methods face the problem of setting boundary conditions (see Ref. [2]).

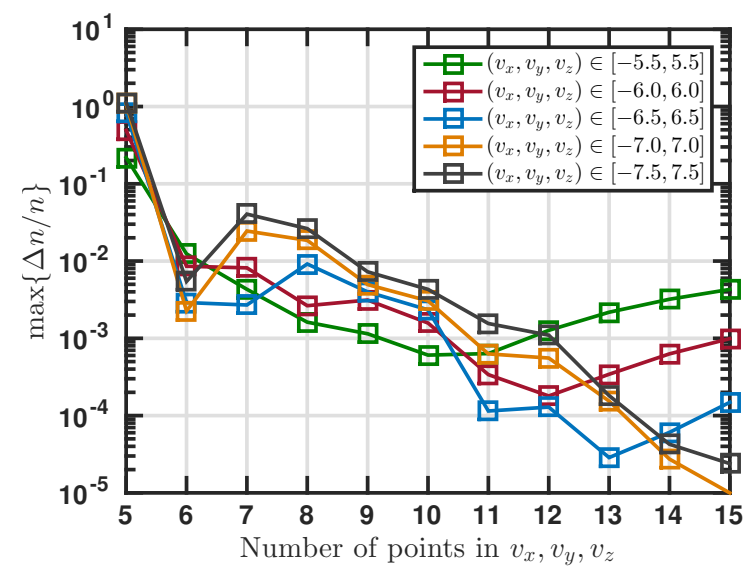

Figure 5: Conservation of density in the 3D relaxation study while varying the domain covered by the collocation points. Here $\gamma \Delta^{2}=0.5$. Energy and momentum behave similarly.

\section{Summary}

In this letter, we have derived a completely new approach to collisional dynamics in plasmas. We have shown how to implement a Gaussian radial-basis-function ansatz to discretize the

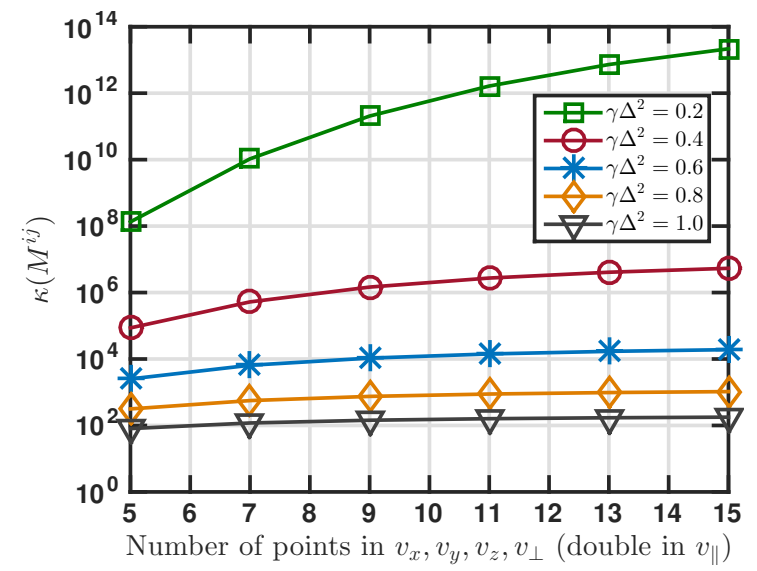

Figure 6: Condition number of the 3D RBF matrix for different values of $\gamma \Delta^{2}$.

velocity space, and demonstrated its capabilities in non-linear Fokker-Planck calculations in both full 3D and axisymmetric $2 \mathrm{D}$ velocity-space. In a wider connection to kinetic theory we have provided also the discretization of the advection terms and given analytical ansatz expressions for all relevant fluidmoments.

In the future, our method could be used to solve various problems where non-equilibrium kinetic effects are important, as long as the Landau-approximation for the collision integral is valid. For example, time-evolution of velocity space loss-cones and holes, often encountered in pedestal physics in magnetic fusion experiments, could be of interest. In addition, the method could be applied to describe how fast particle populations slowdown and deposit energy and momenta to main plasma. We also expect the method to find applications in linear problems.

\section{Acknowledgements}

We are grateful for the encouraging comments and insightful discussions with Prof. Tünde Fülöp, Dr. Istvan Pusztai, and Mr. Adam Stahl. Especially, without the help from Prof. Fülöp, the collaboration between Chalmers University of Technology and General Atomics would not have been possible. Funding for the collaboration was received from the Chalmers Foundation.

\section{References}

[1] M. N. Rosenbluth, W. M. MacDonald, D. L. Judd, Fokker-Planck Equation for an Inverse-Square Force, Phys. Rev. 107 (1957) 1-6. doi:10.1103/ PhysRev.107.1.

[2] M. A. Dorf, R. H. Cohen, M. Dorr, J. Hittinger, T. D. Rognlien, Progress with the cogent edge kinetic code: Implementing the fokker-planck collision operator, Contrib. Plasma Phys. 54 (4-6) (2014) 517-523. doi : 10.1002/ctpp. 201410023.

[3] A. Pataki, L. Greengard, Fast elliptic solvers in cylindrical coordinates and the coulomb collision operator, J. Comput. Phys. 230 (21) (2011) 7840 7852. doi:10.1016/j.jcp.2011.07.005.

[4] L. Pareschi, G. Russo, G. Toscani, Fast spectral methods for the FokkerPlanck-Landau collision operator, J. Comput. Phys. 165 (1) (2000) 216236. doi:10.1006/jcph.2000.6612. 
[5] J. Park, I. W. Sandberg, Universal approximation using radial-basisfunction networks, Neural Comput. 3 (2) (1991) 246-257. doi:10.1162/ neco.1991.3.2.246.

[6] Z. Xiong, R. Cohen, T. Rognlien, X. Xu, A high-order finite-volume algorithm for fokker-planck collisions in magnetized plasmas, J. Comput. Phys. 227 (15) (2008) 7192 - 7205. doi:10.1016/j.jcp. 2008. 04.004.

[7] B. Fornberg, E. Larsson, N. Flyer, Stable computations with gaussian radial basis functions, SIAM Journal on Scientific Computing 33 (2) (2011) 869892. doi:10.1137/09076756X. 